\title{
聚丙烯酸钠和丝素蛋白自膨胀水凝胶的制备及其释药性能
}

\author{
马晓晔 $a$ 施丽君 $b, c$ 周 涓*, $b$ 朱 君 ${ }^{b}$ 钟 建 ${ }^{b}$ 魏锐利 $*, a$ \\ ( ${ }^{a}$ 第二军医大学附属长征医院眼科 上海 200003) \\ ( ${ }^{b}$ 纳米技术及应用国家工程研究中心 上海 200241) \\ ( 上海交通大学材料学院 上海 200240 )
}

\begin{abstract}
摘要 通过自由基聚合的方法合成制备了自膨胀 PAAS-SF semi-IPN 水凝胶(聚丙烯酸钠-丝素半互穿网络水凝胶), 并 进一步探讨其作为微创治疗中组织植入物的潜能. 主要研究了 $\mathrm{SF}$ (丝素蛋白)和 AA(丙烯酸)的重量比对 PAAS-SF semi-IPN 水凝胶的结构、膨胀性以及力学性能的影响. 此外, 我们还研究了抗生素药物阿莫西林(AMO)在 PAAS-SF semi-IPN 水凝胶中的包载与释放性能. 结果表明, 随着水凝胶中 $\mathrm{SF}$ 含量的增加, 膨胀率增大, 压缩强度减小, 药物释 放速率加快, 且 PAAS-SF20 semi-IPN 水凝胶在 $120 \mathrm{~h}$ 内能释放 $(83.4 \pm 0.9) \%$ 的药物. 所开发的自膨胀 PAAS-SF semi-IPN 水凝胶具有高膨胀率和良好的药物释放能力, 将在药物输送或其它植入材料领域有良好的应用前景.
\end{abstract}

关键词 自膨胀; 丝素蛋白; 聚丙烯酸钠; 膨胀率; 药物释放

\section{Preparation of the Self-inflating Hydrogels Based on Poly(sodium acrylate) and Silk Fibroin and Their Drug Releasing Properties}

\author{
Ma, Xiaoye ${ }^{a} \quad$ Shi, Lijun ${ }^{b, c} \quad$ Zhou, Juan $^{*, b} \quad$ Zhu, Jun $^{b} \quad$ Zhong, Jian $^{b} \quad$ Wei, Ruili ${ }^{*, a}$ \\ ( ${ }^{a}$ Department of Ophthalmology, Changzheng Hospital, Second Military Medical University, Shanghai 200003) \\ ( ${ }^{b}$ National Engineering Research Center for Nanotechnology, Shanghai 200241) \\ ( ${ }^{c}$ College of Materials Science and Engineering, Shanghai Jiao Tong University, Shanghai 200240)
}

\begin{abstract}
A self-inflating hydrogel based on silk fibroin (SF) and poly(sodium acrylate) (PAAS) was synthesized by the free radical copolymerization. Furthermore, its potential as a tissue implant with minimally invasive treatment was investigated. The effects of the weight ratio of SF to AA (acrylic acid) on the structure, swelling properties and mechanical properties of the PAAS-SF semi-IPN hydrogels were investigated. In addition, the loading and release of amoxicillin of the PAAS-SF semi-IPN hydrogels were studied. With increasing SF content in the hydrogels, the swelling ratio and the rate of drug release increased, while the compressive strength decreased. It was observed that $(83.4 \pm 0.9) \%$ of the loaded drug was released within $120 \mathrm{~h}$ for the PAAS-SF20 semi-IPN hydrogels. The results revealed that the self-inflating PAAS-SF semi-IPN hydrogels with high swelling ratio and good drug releasing capabilities may have potential in the drug delivery or other implantable materials.
\end{abstract}

Keywords self-inflating; silk fibroin; poly(sodium acrylate); swelling ratio; drug release

传统的组织重建手术会引起较大的创伤，造成并发 症，使患者遭受巨大的痛苦，且需要经历长时间的恢复. 相反，微创治疗能极大程度地缓解这些症状，已吸引了 越来越多的关注. 作为可用于微创治疗的一种材料, 水 凝胶由亲水性聚合物链的三维(3D)网络形成, 在水环境 下会发生膨胀，但不会溶解 ${ }^{[1 \sim 4]}$. 其中，自膨胀水凝胶 在微创治疗中有着潜在的应用 ${ }^{[5 \sim 7]}$, 其主要通过渗透作
用吸水，在干燥状态下体积较小，可用小直径注射器将 其注射到特定位置，避免造成大的创伤. 它可用于各种 组织的填充或者缺损修复，如皮下组织填充、眼眀植入 物、软骨修复等，是组织重建技术的重要进步. 此外，在 一般情况下，手术后为防止伤口感染，常通过口服或者 肌肉注射的方法使用抗生素药物, 这样会造成药物在局 部浓度低，导致药效不佳. 而将药物载入植入材料中，

\footnotetext{
*E-mail: ruiliwei@gmail.com; xmcjuan@gmail.com

Received December 13, 2012; revised December 20, 2012; published online January 4, 2013.

Project supported by the Army Medical Technology "12th Five-Year Plan" Scientific Research Projects of China (Surface Project, CWS11J131) and the Natural Science Foundation of Shanghai (11ZR1425200).

全军医学科技 “十二五”科研(面上项目, No. CWS11J131)和上海市自然科学基金(No. 11ZR1425200)资助项目.
} 
就能在感染部位提供局部较高的药物浓度, 使药物的药 效提高, 并能降低全身毒性 $\left.{ }^{[8} 10\right]$. 因此, 如果能将自膨 胀凝胶作为载药系统, 则载药自膨胀水凝胶不仅具有微 创的特性, 且能在局部释放药物, 作为新型生物材料具 有较大的应用前景. 然而, 关于载药自膨胀水凝胶的研 究目前还少有报道.

互穿网络(IPN)和半互穿网络(semi-IPN)凝胶由于存 在两种聚合物的协同作用, 具有较好的机械强度和载药 性能 ${ }^{[11,12]}$. 此外, IPN 和 semi-IPN 水凝胶还具有载药、 可控药物释放以及手术创伤小的优点, 是一种有前途和 多功能的植入材料, 在药物输送系统的应用具有很大的 潜力. 目前, 许多天然与人工合成聚合物已被广泛用于 制备 IPN 或 semi-IPN 水凝胶, 其优势在于能同时结合合 成聚合物优异的力学性能与天然材料的仿生性能 ${ }^{[13]}$. 例如, 作为一种人工合成聚合物, 聚丙烯酸(PAA)或聚 丙烯酸钠 (PAAS) 的高吸水性水凝胶在药物输送领域引 起了广泛关注, 在生物医学材料领域表现出极大的潜在 应用价值 ${ }^{[14 ~ 19]}$. Changez 等 ${ }^{[14 ~ 16]}$ 制备了聚丙烯酸和明胶 复合的 semi-IPN 水凝胶. 结果表明, 该复合凝胶具有好 的药物包载及释放性能, 有望用于治疗局部慢性细菌或 真菌感染. 另外, 作为一种常见的天然高分子, 丝素蛋 白(SF)由于其独特的力学性能和良好的生物相容性, 在 组织替代材料或药物控释领域均具有广泛的应用, 其应 用形式多样, 包括三维支架、水凝胶、静电纺丝布、微

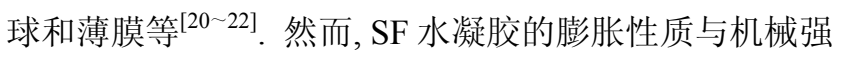
度不佳限制了其在生物医学材料中的应用. 但是通过将 $\mathrm{SF}$ 和其它聚合物组成 semi-IPN 水凝胶, 能改善 SF 水凝 胶的膨胀性质和机械性能 23225$]$.

本文的主要目的是为组织重建提供新型自膨胀凝 胶材料, 使其具有微创治疗和局部药物释放的优点. 通 过结合 SF 的生物相容性和 PAAS 的特殊膨胀性能的优 势, 我们制备了 PAAS-SF semi-IPN 水凝胶. 我们研究 了组成结构对水凝胶溶胀比和体积膨胀率的影响; 进一 步地, 我们研究了该水凝胶的体外药物包载能力和药物 释放动力学, 以评估其作为具有药物释放能力的植入物 的潜力.

\section{1 结果与讨论}

\section{1 自膨胀 PAAS-SF semi-IPN 水凝胶的制备}

通过混合不同比例的 SF 溶液和 $\mathrm{AA}$ (丙烯酸)溶液, 我们制备得到了 PAAS-SF semi-IPN 水凝胶, 图 1a 所示 为 PAAS- SF semi-IPN 水凝胶的形成过程. 根据 SF 与 AA 的质量比为 $0 / 100,10 / 100$ 和 20/100, 将所得凝胶分 别标记为 PAAS、PAAS-SF10 和 PAAS-SF20. 聚合过程 在温度为 $60{ }^{\circ} \mathrm{C}$ 时发生, 过硫酸根离子受热分解产生自
由基，引发 $\mathrm{AA}$ 的聚合，在交联剂 $N, N^{\prime}$-亚甲基双丙烯酰 胺(MBA)的存在下, 发生交联反应, 最终得到具有三维 网状结构的 PAAS 水凝胶. SF 分子链扩散并且缠结于 PAAS 三维网络中. 所制备的凝胶进行纯化, 以除去残 留的化学品, 我们发现所有的水凝胶均有约 $1.3 \%$ 的质 量损失，换言之，水凝胶的产率约为 $98.7 \%$.

不同组分的 PAAS-SF semi-IPN 水凝胶具有不同的 色泽, 如图 $1 \mathrm{~b}$ 所示. 干燥的水凝胶较硬, 在生理盐水 (PSW, $0.9 \% \mathrm{NaCl}$ 水溶液)和阿莫西林 $(\mathrm{AMO})$ 溶液溶胀之 后，水凝胶明显膨胀，且富有弹性。随着 PAAS-SF semi-IPN 水凝胶中 SF 含量的增加, 水凝胶在干燥状态 时的颜色从较透明的白色变为不透明的微黄色，而水凝 胶溶胀后由透明变为半透明. 颜色的变化说明 SF 已成 功地缠结在 PAAS 三维网络中, 证明了通过自由基聚合 制备自膨胀 PAAS-SF semi-IPN 水凝胶的可行性.

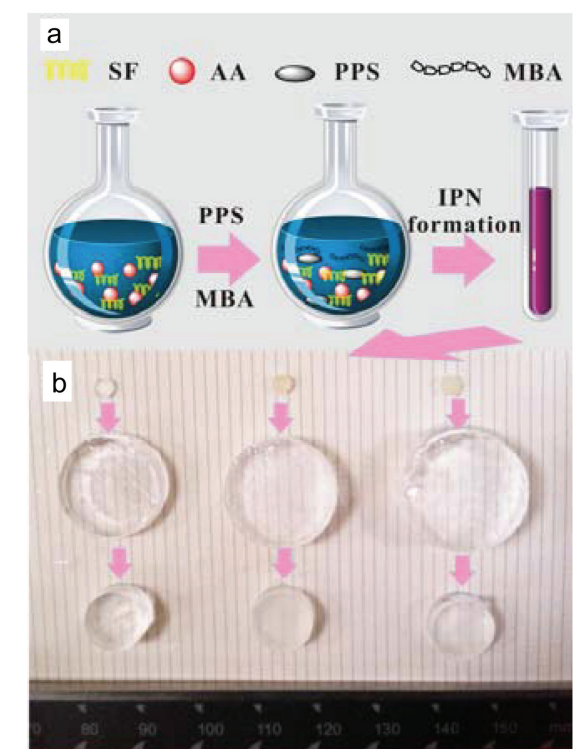

图 1 (a) PAAS-SF semi-IPN 水凝胶形成的示意图; (b)不同 SF 组分的 PAAS-SF semi-IPN 水凝胶在脱模后的宏观图

Figure 1 (a) Schematic representation of formation of the PAAS-SF semi-IPN hydrogels, and (b) typical appearance of different PAAS-SF semi-IPN hydrogels after removing from moulds

The hydrogels from left to right are the PAAS, PAAS-SF10, and PAAS-SF20 semi-IPN hydrogels, respectively. From up to down, the hydrogels represent the dried hydrogels, the swollen hydrogels after swelling in water for $24 \mathrm{~h}$, and the swollen hydrogel after swelling in PSW for $24 \mathrm{~h}$, respectively

\section{2 自膨胀 PAAS-SF semi-IPN 水凝胶的结构分析和 热分析}

图 2 所示为冷冻干燥 SF, PAAS 和不同组分的 PAAS-SF semi-IPN 水凝胶的 FTIR 图谱, 光谱范围为 $2000 \sim 800 \mathrm{~cm}^{-1}$. 冷冻干燥 SF 的 FTIR 图谱显示在 1641, 1514 和 $1230 \mathrm{~cm}^{-1}$ 处有典型的吸收峰, 分别对应于酰 
胺- $\mathrm{I}(\mathrm{C}=\mathrm{O}$ 伸缩振动, 无规卷曲), 酰胺-II $(\mathrm{N}-\mathrm{H}$ 弯曲 振动以及 $\mathrm{C}-\mathrm{N}$ 伸缩振动, $\beta$-折叠) 和酰胺- $\mathrm{III}(\mathrm{N}-\mathrm{H}$ 变形 和 $\mathrm{C}-\mathrm{N}$ 伸缩振动, $\beta$-折叠) 的特征频带 ${ }^{[26,27]}$.

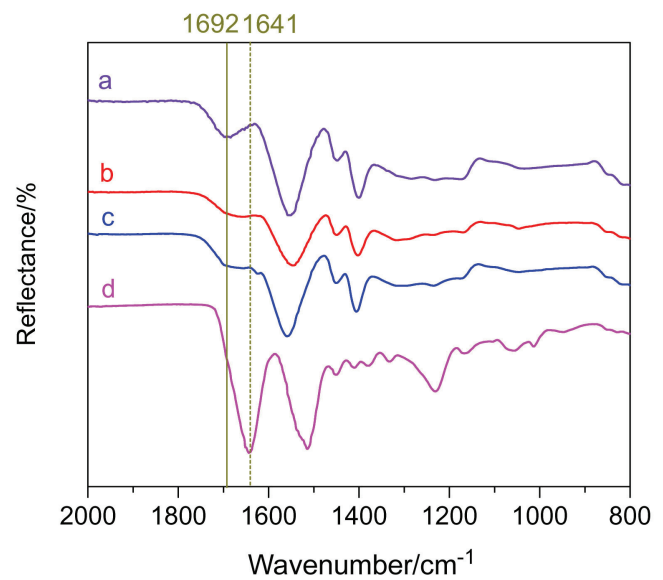

图 2 PAAS、冷冻干燥 $\mathrm{SF}$ 和不同 SF 含量的 PAAS-SF semi-IPN 水凝胶的 FTIR 光谱图

Figure 2 FTIR of the PAAS, freeze dried SF and PAAS-SF semi-IPN hydrogels with different $\mathrm{SF}$ concentration

(a) PAAS; (b) PAAS-SF10; (c) PAAS-SF20; (d) freeze dried 10\% SF

在PAAS-SF10 semi-IPN 水凝胶的 FTIR 图谱中没有 观察到典型的 SF 特征吸收峰, 这可能是由于 $\mathrm{SF}$ 在水凝 胶中的含量较低, 难以检测出. 从 PAAS-SF20 semi-IPN 水凝胶的红外光谱可以看出, SF 在酰胺 I 带的特征峰发 生了从 1641 到 $1625 \mathrm{~cm}^{-1}$ 的红移. 这一结果表明, 在 PAAS-SF semi-IPN 水凝胶的制备过程中, 随着水凝胶 的形成, $\mathrm{SF}$ 发生了 $\beta$-折叠结构转变. PAAS 水凝胶在 1692 和 $1555 \mathrm{~cm}^{-1}$ 的特征峰, 分别对应于 $\mathrm{C}=\mathrm{O}$ 伸缩振 动和 $\mathrm{COO}^{-}$不对称伸缩振动 ${ }^{[28,29]}$. 随着 $\mathrm{SF}$ 含量的增加, $1692 \mathrm{~cm}^{-1}$ 处的吸收峰强度下降, 而位于 $1555 \mathrm{~cm}^{-1}$ 处的 特征峰首先红移至 $1544 \mathrm{~cm}^{-1}$ 处(PAAS-SF10 semi-IPN 水凝胶), 然后又蓝移至 $1555 \mathrm{~cm}^{-1}$ 处(PAAS-SF20 semi-IPN 水凝胶). 这些结果表明, SF 和 PAAS 之间存在 分子间相互作用, 且作用模式随着 SF 含量的不同发生 变化.

PAAS, SF 和 PAAS-SF semi-IPN 水凝胶的 TG 图示 于图 3. 由 PAAS 水凝胶的 TG 曲线所知, PAAS 水凝胶 有两个热分解步骤, 其中主要的热分解温度 $\left(T_{\mathrm{d}}\right)$ 在 273 ${ }^{\circ} \mathrm{C}$, 归因于聚丙烯酸钠中结合水的失去. SF 也显示出两 个热分解步骤, 其主要的热分解温度为 $\left(T_{\mathrm{d}}\right) 250{ }^{\circ} \mathrm{C}$. 从 图 3 可以看出, PAAS-SF semi-IPN 水凝胶与 PAAS 水凝 胶有着相似的 TG 曲线, PAAS-SF10 和 PAAS-SF20 semi-IPN 水凝胶的第一个 $T_{\mathrm{d}}$ 分别是 255 和 $275{ }^{\circ} \mathrm{C}$, 这说 明 PAAS-SF10 水凝胶和其它水凝胶相比有较低的 $T_{\mathrm{d}}$ 值 和较差的热稳定性. 而 PAAS-SF20 semi-IPN 水凝胶的
$T_{\mathrm{d}}$ 值较高并且近似于 PAAS 水凝胶, 这与 FTIR 图谱的 规律相似.

\section{3 自膨胀 PAAS-SF semi-IPN 水凝胶的溶胀比和体 积膨胀率}

水凝胶的溶胀特性对水凝胶中小分子的扩散行为 有显著的影响. 我们研究了 $\mathrm{SF}$ 含量对水凝胶溶胀行为 的影响, 包括溶胀比和体积膨胀率. 水凝胶的溶胀比是 指凝胶吸水后的质量变化, 而体积膨胀率则表示凝胶吸 水后体积的变化程度. 在我们之前的研究中已证实, 二 者具有相同的变化趋势但表示意义不同 ${ }^{[30]}$.

图 $4 \mathrm{a}$ 和图 $4 \mathrm{~b}$ 分别表示了 $\mathrm{SF}$ 含量对 PAAS-SF semi-IPN 水凝胶在 $37{ }^{\circ} \mathrm{C}$ 下的水与 PSW 中溶胀动力学的 影响. 由图可见, PAAS-SF semi-IPN 水凝胶的吸水量随 着时间增加而增加, 直到达到平衡状态. 所有水凝胶都 迅速膨胀, 在水中和 PSW 中分别在近 8 和 $12 \mathrm{~h}$ 内达到 平衡, 他们在水中的溶胀比都远高于 150 , 而在 PSW 中 则是高于 28. 此外, PAAS-SF semi-IPN 水凝胶无论在水 中或 PSW 中的溶胀比都要高于 PAAS 水凝胶. PAAS-SF20 semi-IPN 水凝胶表现出最大的溶胀比，在 水中和 PSW 中分别为 180 和 30 . 图 5 为 PAAS-SF semi-IPN 水凝胶在水中和 PSW 中的体积膨胀率. 由图 可见, 所有水凝胶在水中和 PSW 中的体积膨胀率分别 都大于 180 和 25 . 与水凝胶的溶胀比类似, PAAS-SF10 和 PAAS-SF20 无论在水中还是在 PSW 中都比 PAAS 有更高的体积膨胀率, PAAS-SF20 同样拥有最高的体积 膨胀率.

\section{4 自膨胀 PAAS-SF semi-IPN 水凝胶的相互作用机 制}

众所周知, $\mathrm{NH}_{2}$ 和 $\mathrm{COOH}$ 等官能团, 能与 $\mathrm{COO}^{-}$离 子形成强烈的氢键作用. 当 $10 \%$ 的 SF 添加到 PAAS 水 凝胶中, SF 分子和 PAAS 链通过氢键作用，使得聚合物 链密度和聚合物链上的电荷增加 ${ }^{[13]}$, 导致了 $\mathrm{COO}^{-}$非对 称伸缩振动谱带的红移和 PAAS-SF10 水凝胶的分解温 度降低. 然而, 随着 SF 含量的增加, 由于聚合物链上的 电荷增加, 静电斥力增加, 导致聚合物链产生大的空间 位阻 ${ }^{[31]}$. 这可以由 $\mathrm{COO}^{-}$非对称伸缩带的蓝移和 PAASSF20 水凝胶的高热分解温度得到证实.

此外, 增加的聚合物链能导致溶胀比减小, 但这种 减小会被增加的静电哌力削弱. 因此, 随着 SF 含量的 增加, 溶胀比和体积膨胀率都得到增加. 然而, 水凝胶 从干燥状态开始的吸水动力学主要由聚合物链控制, 这 导致了在初始的 $8 \mathrm{~h}$ 内, 随着 SF 含量的增加, 溶胀速率 较慢. 

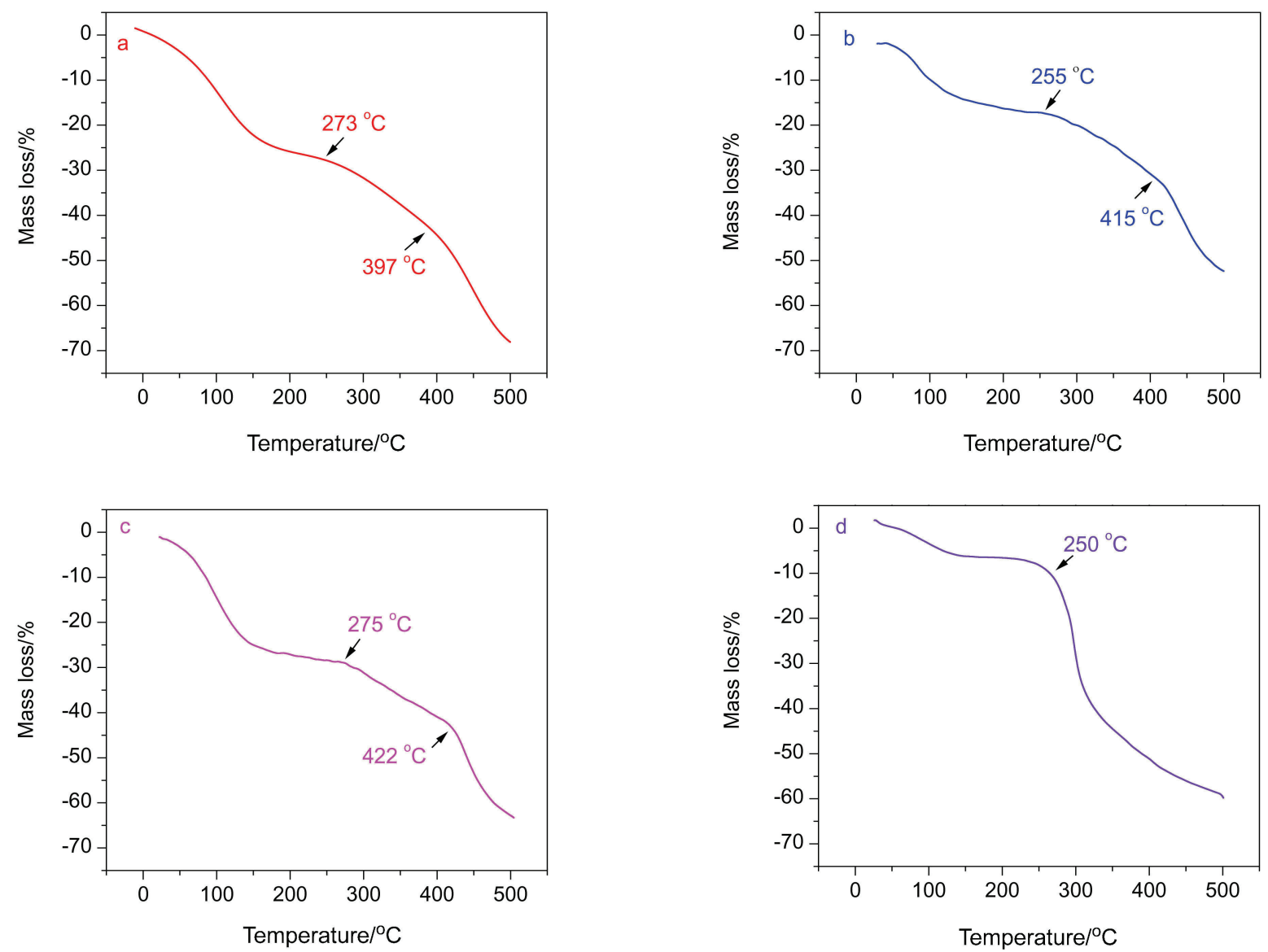

图 3 PAAS、SF 和不同 SF 含量的 PAAS-SF semi-IPN 水凝胶的 TG 图

Figure 3 TG of the PAAS, SF and PAAS-SF semi-IPN hydrogels with different SF concentration (a) PAAS; (b) PAAS-SF10; (c) PAAS-SF20; (d) freeze dried $10 \% \mathrm{SF}$
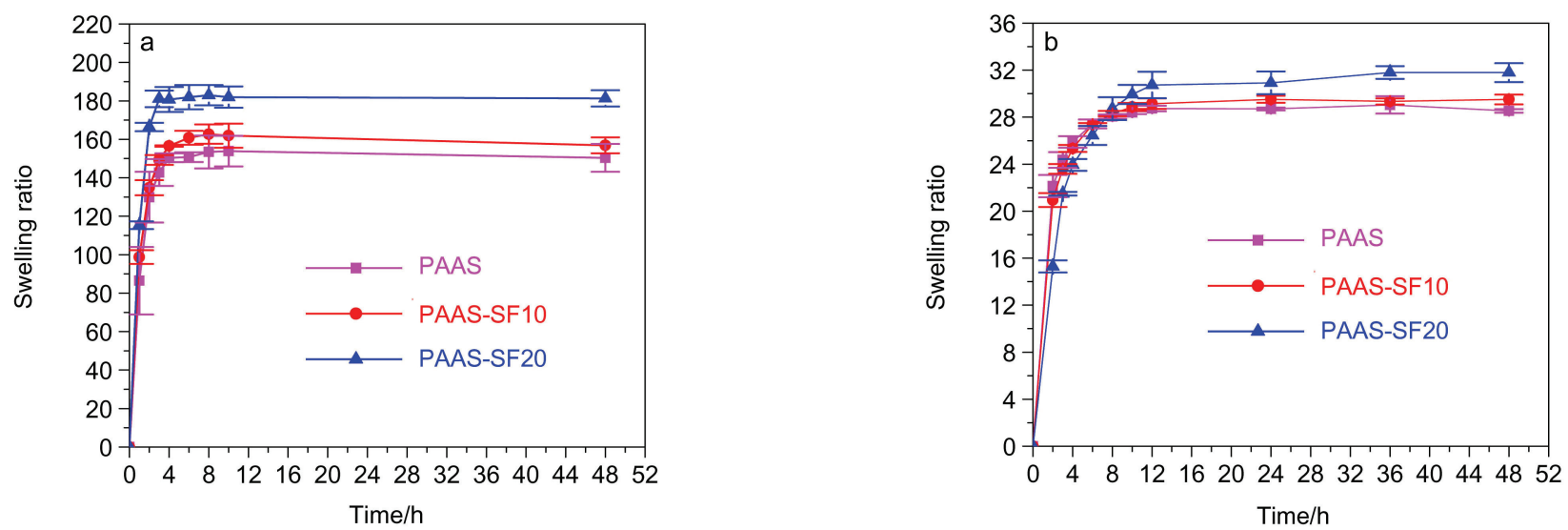

图 4 (a) PAAS-SF semi-IPN 水凝胶在水中的溶胀动力学及(b) PAAS-SF semi-IPN 水凝胶在 PSW 中的溶胀动力学

Figure 4 (a) Swelling kinetics of the PAAS-SF semi-IPN hydrogels in water, and (b) swelling kinetics of the PAAS-SF semi-IPN hydrogels in PSW

\section{5 自膨胀 PAAS-SF semi-IPN 水凝胶的力学性能}

用于药物持续释放的 semi-IPN 水凝胶需要在生理 条件下具有一定的力学强度, 以抵抗体内的各种应力. 表 1 显示了各组分的 semi-IPN 水凝胶的压缩强度值和 失效时的变形量. 结果表明, 随着 SF 含量的增加, 压缩
强度下降, 失效时的变形减小. 其原因在于: 随着 $\mathrm{SF}$ 含 量的增加, PAAS-SF semi-IPN 水凝胶的溶胀比增加, 这 导致了它的水含量增加, 刚性增强. 另外, PAAS 有着明 显高于 PAAS-SF semi-IPN 水凝胶的压缩强度. 这是由 于将 SF 引入到 PAAS 水凝胶中可能导致应力集中点的 


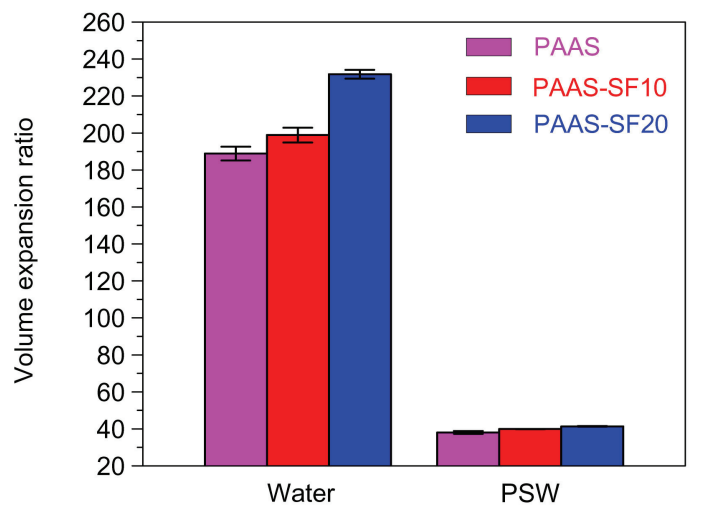

图 5 PAAS-SF semi-IPN 水凝胶在水和 PSW 中的体积膨胀率 Figure 5 Volume expansion ratios of the PAAS-SF semi-IPN hydrogels in water and PSW

出现.

表 1 不同组分的 PAAS-SF semi-IPN 水凝胶的机械性能

Table 1 Mechanical properties of the PAAS-SF semi-IPN hydrogels with different composition.

\begin{tabular}{ccc}
\hline 样品 & 压缩强度 $/ \mathrm{kPa}$ & 失效变形量 $/ \%$ \\
\hline PAAS & $249 \pm 20$ & $67 \pm 3$ \\
PAAS-SF10 & $117 \pm 1$ & $64 \pm 5$ \\
PAAS-SF20 & $115 \pm 5$ & $63 \pm 3$ \\
\hline
\end{tabular}

\section{6 自膨胀 PAAS-SF semi-IPN 水凝胶的载药性能}

临床实践中, 采用抗生素以治疗和预防感染是非常 普遍的, 在植入物中直接负载抗生素, 能在感染组织部 位产生高的抗生素浓度, 并能减少全身毒性. 阿莫西林 (AMO)作为常规和代表性的水溶性抗生素, 已经被广泛 用作模型药物进行研究 ${ }^{[32,33]}$. 我们选用 $\mathrm{AMO}$ 为模型药 物, 探讨了 PAAS-SF 水凝胶作为药物载体的可能性, 研 究了其药物释放效果. 图 6 为包载了 AMO 的 PAAS 和 PAAS-SF semi-IPN 水凝胶的断面 SEM 照片. 由图可见, AMO 在 PAAS 水凝胶和 PAAS-SF semi-IPN 水凝胶中分 布均匀. 随着 SF 含量的增加, 水凝胶的表面形貌从光 滑变为粗糙. 相比 PAAS-SF20 semi-IPN 水凝胶, PAASSF10 semi-IPN 水凝胶表面形貌更粗粘, 在其表面有大 量的突起. 这是由于 PAAS 和 SF 之间存在较强的静电 相互作用, 造成相分离, 最终导致粗粘的表面.

另外, 我们研究了 PAAS-SF semi-IPN 水凝胶的组 分对其在 AMO 溶液中的溶胀比和 AMO 在 PAAS-SF semi-IPN 水凝胶中载药量的影响. 如图 7a 所示, 随着 SF 含量的增加, PAAS-SF semi-IPN 水凝胶在 AMO 溶液 中的溶胀比略有增加, 这与在 PSW 中的情况相似. 此 外, 如图 7b 所示, 水凝胶的载药量同样随着 $\mathrm{SF}$ 含量的 增加略有增加. PAAS-SF20 semi-IPN 水凝胶具有最大的 溶胀比和最大的载药量, 分别为 $(33.1 \pm 2.1) \%,(39.0 \pm$ $1.2) \%$. 由于 PAAS-SF semi-IPN 水凝胶的溶胀规律与载
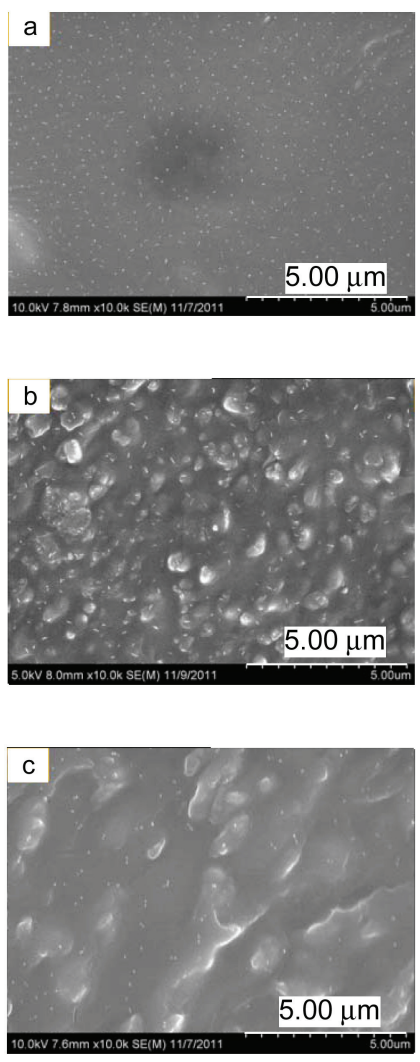

图 6 不同组分的 PAAS-SF semi-IPN 水凝胶包载了 AMO 后 的 SEM 断面照片

Figure 6 SEM photographs showing cross-sectional morphology within AMO loaded PAAS-SF semi-IPN hydrogels with different composition

(a) PAAS; (b) PAAS-SF10; (c) PAAS- SF20; scale bars $=5 \mu \mathrm{m}$

药规律相似, 我们推测凝胶的载药主要是通过物理吸 附，当凝胶发生溶胀时，药物就会随着水的渗透同时进 入到 PAAS-SF semi-IPN 水凝胶中.

\section{7 自膨胀 PAAS-SF semi-IPN 水凝胶在 PSW 和水 中的药物释放动力学}

我们进一步研究了凝胶的药物释放动力学, 图 8 为 不同组分的 PAAS-SF semi-IPN 水凝胶的药物释放曲线. 由图可见, 所有的水凝胶在 $1 \mathrm{~h}$ 都能观察到明显的初期 突释现象, PAAS-SF10 和 PAAS-SF20semi-IPN 水凝胶分 别具有 $40 \%$ 和 $58 \%$ 的突释药量, 其中, PAAS-SF 20 semi-IPN 水凝胶有最大的突释量. PAAS, PAAS-SF10 和 PAAS-SF20 semi-IPN 水凝胶在 $120 \mathrm{~h}$ 的最终药物释放量 分别为 $(79.9 \pm 0.6) \%,(82.6 \pm 0.3) \%$ 和 $(83.4 \pm 0.9) \%$. PAAS-SF semi-IPN 水凝胶在初期释放可以归因于表面 吸附药物的释放. PAAS-SF20 semi-IPN 水凝胶有最大的 突释量和最终释放量, 这主要是因为它有最大的载药量 和高的溶胀能力 ${ }^{[34]}$. 这一结果表明, PAAS-SF semi-IPN 水凝胶的组分对其释药性具有一定影响. 由于所制备的 PAAS-SF semi-IPN 水凝胶拥有快速药物释放的特性, 

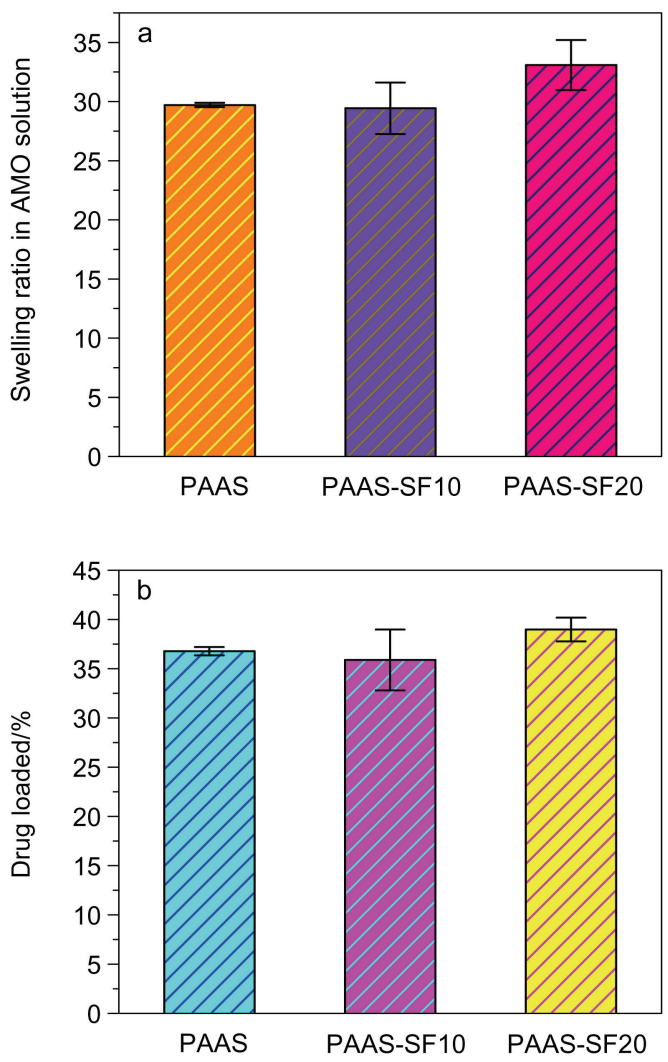

图 7 (a) PAAS-SF semi-IPN 水凝胶在 AMO 中的溶胀比; (b) PAAS-SF semi-IPN 水凝胶的载药量

Figure 7 (a) Swelling ratio of the PAAS-SF semi-IPN hydrogels in AMO solution, and (b) drug loaded content of the PAAS-SF semi-IPN hydrogels

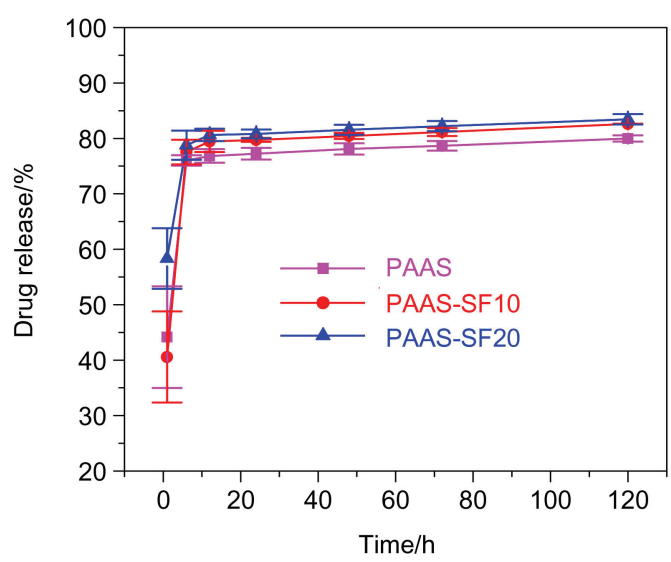

图 8 不同组分 PAAS-SF semi-IPN 水凝胶的药物释放曲线

Figure 8 Drug release of the drug loaded PAAS-SF semi-IPN hydrogels with different composition at different time points

将在组织修复重建领域有很大的应用潜力.

\section{2 结论}

我们成功合成了由 SF 和 PAAS 组成的自膨胀 semiIPN 水凝胶, 研究了组分对水凝胶性质的影响, 采用阿
莫西林(AMO)为药物模型探讨了 PAAS-SF semi-IPN 水 凝胶作为药物载体的可行性, 研究了其药物释放性能. 随着 SF 在水凝胶中含量的增加, PAAS-SF semi-IPN 水 凝胶的溶胀比和载药量都得到增加, 压缩强度从不含 $\mathrm{SF}$ 的 $(249 \pm 20) \mathrm{kPa}$ 减小到 $(115 \pm 5) \mathrm{kPa}$. PAAS-SF20 semi-IPN 水凝胶在生理盐水(PSW)中溶胀比、载药量以 及释药量均为最大，分别为 $(33.1 \pm 2.1) \%,(39.0 \pm 1.2) \%$, 在 $120 \mathrm{~h}$ 内能释放 $(83.4 \pm 0.9) \%$ 的药物. 研究表明，通过 控制自膨胀 PAAS-SF semi-IPN 水凝胶的组分，可以很 容易地控制凝胶的性能如溶胀比、载药量和释药量. 所 制备的自膨胀 PAAS-SF semi-IPN 水凝胶由于具有较高 的膨胀率和较好的载药性能, 可用于组织重建的微创治 疗，并具有局部药物治疗的效果，满足不断增长的特定 组织重建的要求.

\section{3 实验部分}

\section{1 仪器与试剂}

丙烯酸( $\mathrm{AA})$ 、过硫酸钾( $\mathrm{PPS}$, 直接使用) 和 $N, N^{\prime}$-亚 甲基双丙烯酰胺(MBA，直接使用)购于国药集团化学试 剂有限公司, 所有试剂均为分析纯. 用蒸馏水配制 0.1 $\mathrm{mol} \cdot \mathrm{L}^{-1} \mathrm{PPS}$ 和 $0.1 \mathrm{~mol} \cdot \mathrm{L}^{-1} \mathrm{MBA}$, 然后过滤待用. 丙烯 酸(AA)通过减压蒸馏纯化, 纯化后的 $\mathrm{AA}$ 置于 $4{ }^{\circ} \mathrm{C}$ 避光 保存待用. 蚕茧，由中国江苏金源丝绸厂提供. 透析袋 截留分子量为 $8000 \sim 14000$, 产自美国.

\section{2 实验方法}

\subsubsection{SF 水溶液的制备}

$\mathrm{SF}$ 水溶液的制备方法简述如下 ${ }^{[35]}$ : 处理干净的蚕 茧在质量分数为 $0.5 \%$ 的 $\mathrm{Na}_{2} \mathrm{CO}_{3}$ 水溶液中煮沸 $1 \mathrm{~h}$, 用蒸 馏水冲洗干净, 以除去丝胶. 脱胶后的 $\mathrm{SF}$ 在 $80{ }^{\circ} \mathrm{C}$ 下溶 解于 $\mathrm{CaCl}_{2} / \mathrm{H}_{2} \mathrm{O} / \mathrm{C}_{2} \mathrm{H}_{5} \mathrm{OH}$ 溶液(物质的量比为 $1: 8: 2$ ) $40 \mathrm{~min}$, 随后置于透析袋中, 在蒸馏水中透析 $3 \mathrm{~d}$. 再将 溶液取出过滤, 得到初始浓度为 $3 \%$ 的纯净的 $\mathrm{SF}$ 水溶 液. 为了防止丝素溶液的凝胶化转变, 制备的 SF 溶液 在 $4{ }^{\circ} \mathrm{C}$ 下保存待用. $\mathrm{SF}$ 溶液在温度为 $55{ }^{\circ} \mathrm{C}$ 下温和搅拌, 最终得到浓度为 $10 \%$ 的 SF 水溶液. 通过测定丝素溶液 干燥后的固含量来决定丝素溶液的浓度.

\section{2 .1 水凝胶合成}

聚丙烯酸钠(PAAS)水凝胶由自由基引发聚合, PPS 作为引发剂, MBA 作为交联剂. 首先, 质量分数为 $30 \%$ 的 $\mathrm{AA}$ 水溶液在三颈烧瓶中与 $\mathrm{NaOH}(\mathrm{NaOH}$ 与 $\mathrm{AA}$ 的物 质的量比为 $70 \%$ )中和, 并在室温下进行磁力搅拌, 搅拌 过程中持续通入氮气, $10 \mathrm{~min}$ 后, 将与 $\mathrm{AA}$ 质量比为 $0.5 \%$ 的 MBA 和 $0.5 \%$ 的 PPS 溶液逐滴滴入混合溶液中, 再摚拌 $10 \mathrm{~min}$. 然后将混合溶液立即注入模具中, 置于 
干燥箱中, 于 $65{ }^{\circ} \mathrm{C}$ 下反应 $3 \mathrm{~h}$. PAAS-SF semi-IPN 水凝 胶的合成步骤与上述步骤相似, $\mathrm{SF}$ 在 $\mathrm{AA}$ 与 $\mathrm{NaOH}$ 中和 后加入, $\mathrm{SF}$ 与 $\mathrm{AA}$ 的质量分数为 $0 \%, 10 \%$ 和 $20 \%$, 制备 过程示意图如图 1a 所示. 根据 SF 含量, 将 PAAS-SF semi-IPN 水凝胶标记为 PAAS-SF10 和 PAAS-SF20. 反 应完毕后, 将成形的 PAAS 或 PAAS-SF semi-IPN 水凝 胶脱模, 并进一步置于 $65{ }^{\circ} \mathrm{C}$ 下干燥 $24 \mathrm{~h}$. 然后将干燥的 水凝胶浸泡在大量蒸馏水中 $3 \mathrm{~d}$, 每天换水 2 次, 以除去 残留的未反应的化学物质. 在溶胀状态下将水凝胶切割 成圆柱体, 然后在 $60{ }^{\circ} \mathrm{C}$ 下干燥 $24 \mathrm{~h}$, 得到干凝胶.

\section{2 .3 结构及热稳定性}

采用傅立叶变换红外光谱(FTIR, 尼高力 6700)的全 反射附件(ATR)测量 PAAS 和 PAAS-SF semi-IPN 水凝胶 的结构, 在金刚石衬底上积累扫描次数为 16 次, 分辨率 为 $4 \mathrm{~cm}^{-1}$, 记录光谱范围为 $4000 \sim 600 \mathrm{~cm}^{-1}$. 热稳定性 通过林塞斯 STA PT1600 进行检测, 温度范围为 25 $500{ }^{\circ} \mathrm{C}$, 升温速率为 $10{ }^{\circ} \mathrm{C} \cdot \mathrm{min}^{-1}$, 所使用的样品量为 $(10 \pm 5) \mathrm{mg}$. 样品制备过程如下: PAAS 和 PAAS-SF semi-IPN 水凝胶在 $37{ }^{\circ} \mathrm{C}$ 下溶胀 $24 \mathrm{~h}$ 后达到平衡状态, 然后在 $-80{ }^{\circ} \mathrm{C}$ 下冷冻干燥 $48 \mathrm{~h}$. SF 样品由 $10 \%$ 的 $\mathrm{SF}$ 水 溶液在 $-80{ }^{\circ} \mathrm{C}$ 下冷冻干燥 $48 \mathrm{~h}$ 制得.

\section{2 .4 溶胀性能测试}

观察和记录水凝胶从 $0 \sim 48 \mathrm{~h}$ 之间的溶胀行为, 测 定水凝胶在蒸馏水和生理盐水(PSW)中的溶胀比和体积 膨胀率。

溶胀比的计算公式为: 溶胀比 $=m_{\mathrm{s}} / m_{\mathrm{d}}$. 式中, $m_{\mathrm{s}}$ 代 表溶胀后的水凝胶在 $37{ }^{\circ} \mathrm{C}$ 下达到溶胀平衡时的质量, $m_{\mathrm{d}}$ 代表水凝胶在干燥状态下的质量.

体积膨胀率的计算公式为: 体积膨胀率 $=V_{\mathrm{s}} / V_{\mathrm{d}}=$ $\left(D_{\mathrm{s}} / D_{\mathrm{d}}\right)^{3}$. 式中, $V_{\mathrm{s}}$ 和 $D_{\mathrm{s}}$ 分别代表湿水凝胶在 $37{ }^{\circ} \mathrm{C}$ 溶胀 平衡时的体积和直径, $V_{\mathrm{d}}$ 和 $D_{\mathrm{d}}$ 分别代表水凝胶在干燥状 态下的体积和直径 ${ }^{[30]}$. 水凝胶的质量由电子天平测得, 水凝胶的直径由游标卡尺测得, 测试前用滤纸轻轻擦拭 掉水凝胶表面多余的水分. 每个样品测 3 次, 在 ORIGIN 8.0 中采用单因素方差分析方法进行统计分析. 所有的数据以平均值 \pm 标准偏差的形式表示. $p \leqslant 0.05$ 时说明具有统计学意义.

\subsection{5 力学性能}

使用 HY-0230 万能试验机进行水凝胶的力学性能 测定, 力传感器测量精度为 $0.5 \%$, 位移传感器精度为 $0.001 \mathrm{~mm}$, 测试过程中, 样品置于压缩夹具中, 通过上 夹具的移动进行压缩试验, 压缩速率为 $5 \mathrm{~mm} / \mathrm{min}$, 最后 得到凝胶的压缩应力一应变曲线. 测试前样品的制备方 法如下: 圆柱状水凝胶样品在测试前于 PSW 中溶胀 24 $\mathrm{h}$, 样品初始直径和高度均约为 $10 \mathrm{~mm}$. 每种样品测量 4
个平行样, 在 ORIGIN 8.0 中采用单因素方差分析方法 进行统计分析, 所得值以平均值士标准偏差表示.

\subsection{6 阿莫西林在水凝胶中的包载}

将已知质量的水凝胶浸泡于阿莫西林 (AMO) 的 PSW 溶液中(浓度为 $12 \mathrm{mg} / \mathrm{mL}$ ), 在 $37{ }^{\circ} \mathrm{C}$ 下浸泡 $24 \mathrm{~h}$. 取出溶胀后的样品并在 $-80{ }^{\circ} \mathrm{C}$ 下冷冻干燥 $48 \mathrm{~h}$. 然后 冷冻干燥样品在液氮中脆断. 样品断面喷金 $20 \mathrm{~s}$ 后, 通 过扫描电子显微镜(SEM, 日立 S-4800) 观察载药后 PAAS-SF semi-IPN 水凝胶的微观形貌.

取出溶胀后的样品, $65{ }^{\circ} \mathrm{C}$ 下干燥并称重，测定凝胶 的载药量, 按以下公式进行计算: 载药量 $=\left(m_{1}-m_{\mathrm{d}}\right) / m_{\mathrm{d}}$. 式中, $m_{1}$ 代表载药后的干燥水凝胶质量, $m_{\mathrm{d}}$ 代表原始干 燥水凝胶的质量.

经证实，在 PSW 中没有观察到水凝胶质量的改变， 因此凝胶在阿莫西林 $(\mathrm{AMO})$ 的 PSW 溶液中增加的质量 即为药物的负载量.

\subsection{7 阿莫西林在水凝胶中的释放}

取约 $10 \mathrm{mg}$ 载药水凝胶浸泡在 $10 \mathrm{~mL}$ PSW 中, 并置 于 $37{ }^{\circ} \mathrm{C}$ 恒温培养摇床中, 振荡速率为 $50 \mathrm{r} / \mathrm{min}$. 在预先 选定的时间点 $(1,6,12,24,48,72$ 和 $120 \mathrm{~h})$, 取出 $5 \mathrm{~mL}$ 溶液, 使用紫外-可见分光光度计(岛津, UV mini-1240) 检测其在 $273.5 \mathrm{~nm}$ 处的吸收强度, 通过 AMO 的标准曲 线计算溶液中 $\mathrm{AMO}$ 的浓度, 以测定 $\mathrm{AMO}$ 的药物释放 性能. 每次采样后, 溶液中都加入 $5 \mathrm{~mL}$ 新配的 PSW, 以维持总体积恒定. 每种样品取四个平行样进行测试, 数据表示为平均值士标准偏差.

\section{References}

[1] Kopecek, J. Biomaterials 2007, 28, 5185.

[2] Kim, J.; Peng, C.-C.; Chauhan, A. J. Controlled Release 2010, 148, 110.

[3] Sun, J.; Wang, Y.; Dou, S. Chin. Chem. Lett. 2012, 23, 97.

[4] Vashist, A.; Gupta, Y. K.; Ahmad, S. Carbohydr. Polym. 2011, 87, 1433.

[5] Schittkowski, M. P.; Guthoff, R. F. Br. J. Ophthalmol. 2006, 90, 1173.

[6] Kustner, M.; Riebeling, P.; Podmelle, F.; Kaduk, W.; Tost, F. Ophthalmologe 2009, 106, 1126.

[7] Swan, M. C.; Bucknall, D. G.; Goodacre, T. E. E.; Czernuszka, J. T. Acta Biomater. 2011, 7, 1126.

[8] Pescosolido, L.; Vermonden, T.; Malda, J.; Censi, R.; Dhert, W. J. A.; Alhaique, F.; Hennink, W. E.; Matricardi, P. Acta Biomater. 2011, 7, 1627.

[9] Wen, X.; Cao, X. L.; Yin, Z. H.; Wang, T.; Zhao, C. S. Carbohydr. Polym. 2009, 78, 193.

[10] Patel, R. B.; Solorio, L.; Wu, H.; Krupka, T.; Exner, A. A. J. Controlled Release 2010, 147, 350 .

[11] Myung, D.; Koh, W.; Ko, J.; Hu, Y.; Carrasco, M.; Noolandi, J.; Ta, C. N.; Frank, C. W. Polymer 2007, 48, 5376.

[12] Yin, L.; Ding, J.; Fei, L.; He, M.; Cui, F.; Tang, C.; Yin, C. Int. J. Pharm. 2008, 350, 220. 
[13] Mandal, B. B.; Kapoor, S.; Kundu, S. C. Biomaterials 2009, 30, 2826.

[14] Changez, M.; Burugapalli, K.; Koul, V.; Choudhary, V. Biomaterials 2003, 24, 527.

[15] Changez, M.; Koul, V.; Dinda, A. K. Biomaterials 2005, 26, 2095.

[16] Changez, M.; Koul, V.; Krishna, B.; Dinda, A. K.; Choudhary, V. Biomaterials 2004, 25, 139.

[17] Chang, C.; Duan, B.; Cai, J.; Zhang, L. Eur. Polym. J. 2010, 46, 92.

[18] de la Torre, P. M.; Torrado, S.; Torrado, S. Biomaterials 2003, 24, 1459.

[19] Rasool, N.; Yasin, T.; Heng, J. Y. Y.; Akhter, Z. Polymer 2010, 51, 1687.

[20] Vepari, C.; Kaplan, D. L. Prog. Polym. Sci. 2007, 32, 991.

[21] Li, C. M.; Vepari, C.; Jin, H. J.; Kim, H. J.; Kaplan, D. L. Biomaterials 2006, 27, 3115.

[22] Wenk, E.; Merkle, H. P.; Meinel, L. J. Controlled Release 2011, $150,128$.

[23] Kim, S. H.; Nam, Y. S.; Lee, T. S.; Park, W. H. Polym. J. 2003, 35, 185.
[24] Kundu, J.; Poole-Warren, L. A.; Martens, P.; Kundu, S. C. Acta Biomater. 2012, 8, 1720.

[25] Kweon, H.; Yeo, J.-H.; Lee, K.-G.; Lee, H. C.; Na, H. S.; Won, Y. H.; Cho, C. S. Biomed. Mater. 2008, 3, 034115.

[26] Hu, X.; Kaplan, D.; Cebe, P. Macromolecules 2008, 41, 3939.

[27] Teramoto, H.; Miyazawa, M. Biomacromolecules 2005, 6, 2049.

[28] Zhou, W.; Zhang, Y.; Jin, K.; Qiu, X.; Ren, X.; Hu, S.; Zhang, F. J. Appl. Polym. Sci. 2009, 114, 2828.

[29] Lu, Y.; Wang, D.; Li, T.; Zhao, X.; Cao, Y.; Yang, H.; Duan, Y. Y. Biomaterials 2009, 30, 4143.

[30] Ma, X.; Wei, R.; Cheng, J.; Cai, J.; Zhou, J. Carbohydr. Polym. 2011, 86, 313.

[31] Li, X.; Wu, W.; Wang, J.; Duan, Y. Carbohydr. Polym. 2006, 66, 473.

[32] Xu, Q.; Czernuszka, J. T. J. Controlled Release 2008, 127, 146.

[33] Torre, P. M.; Enobakhare, Y.; Torrado, G.; Torrado, S. Biomaterials 2003, 24, 1499.

[34] Lee, K. Y.; Mooney, D. J. Chem. Rev. 2001, 101, 1869.

[35] Zhou, J.; Cao, C.; Ma, X. Int. J. Biol. Macromol. 2009, 45, 504.

(Li, L.; Fan, Y.) 